\title{
Highly Efficient Multigram Synthesis of Dibenzoazacyclooctyne (DBCO) Without Chromatography
}

Stuart A. McNelles, Julia L. Pantaleo, Alex Adronov

Department of Chemistry \& Chemical Biology, McMaster University, 1280 Main Street West, Hamilton, Ontario, Canada, L8S 4M1

\section{Supporting Information}

${ }^{1} \mathrm{H}$ NMR spectra and HPLC traces for the products synthesized.

\section{H NMR Spectra}
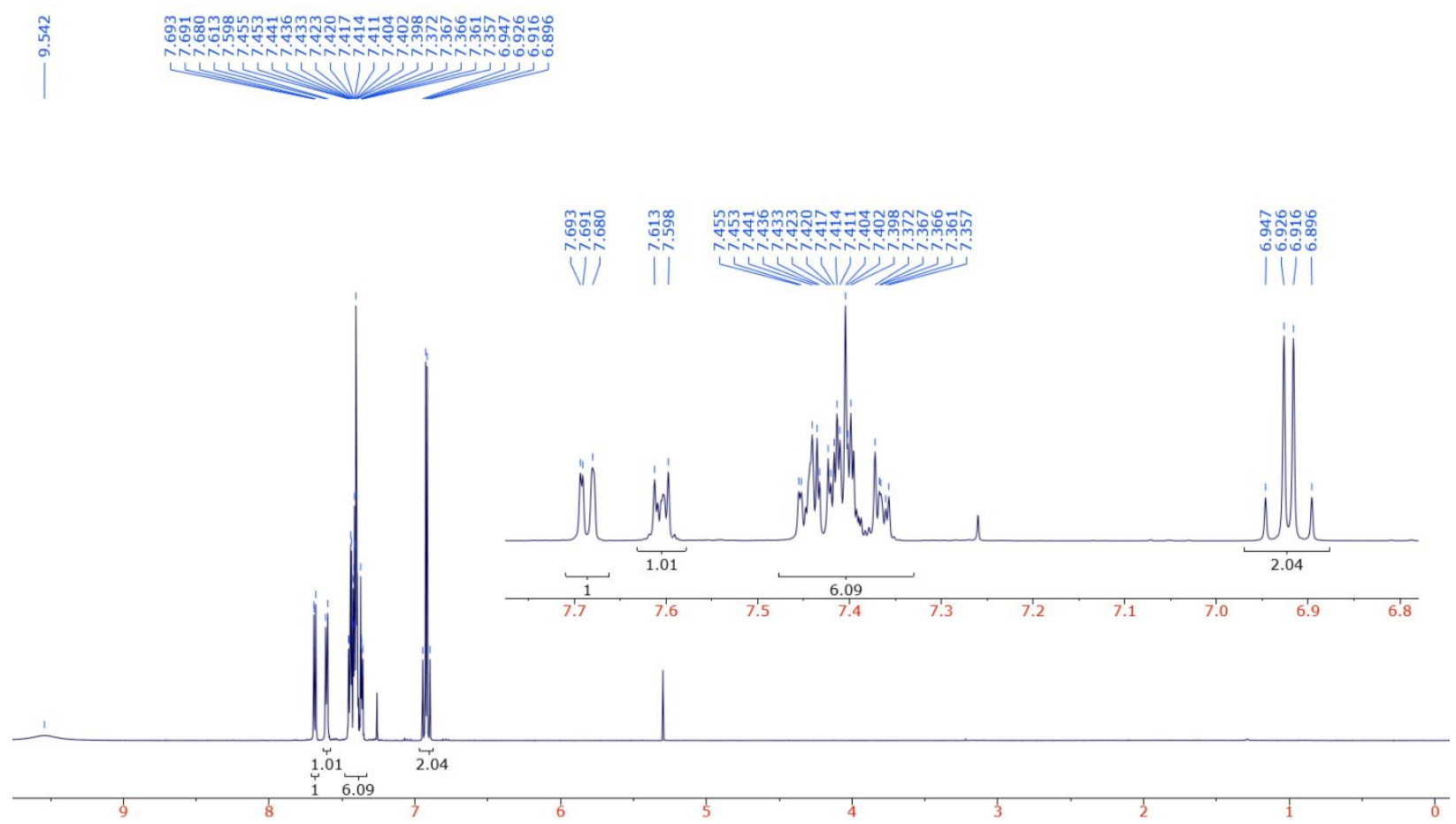

Figure S1. ${ }^{1} \mathrm{H}$ NMR spectrum of Compound 2 in $\mathrm{CDCl}_{3}$ 


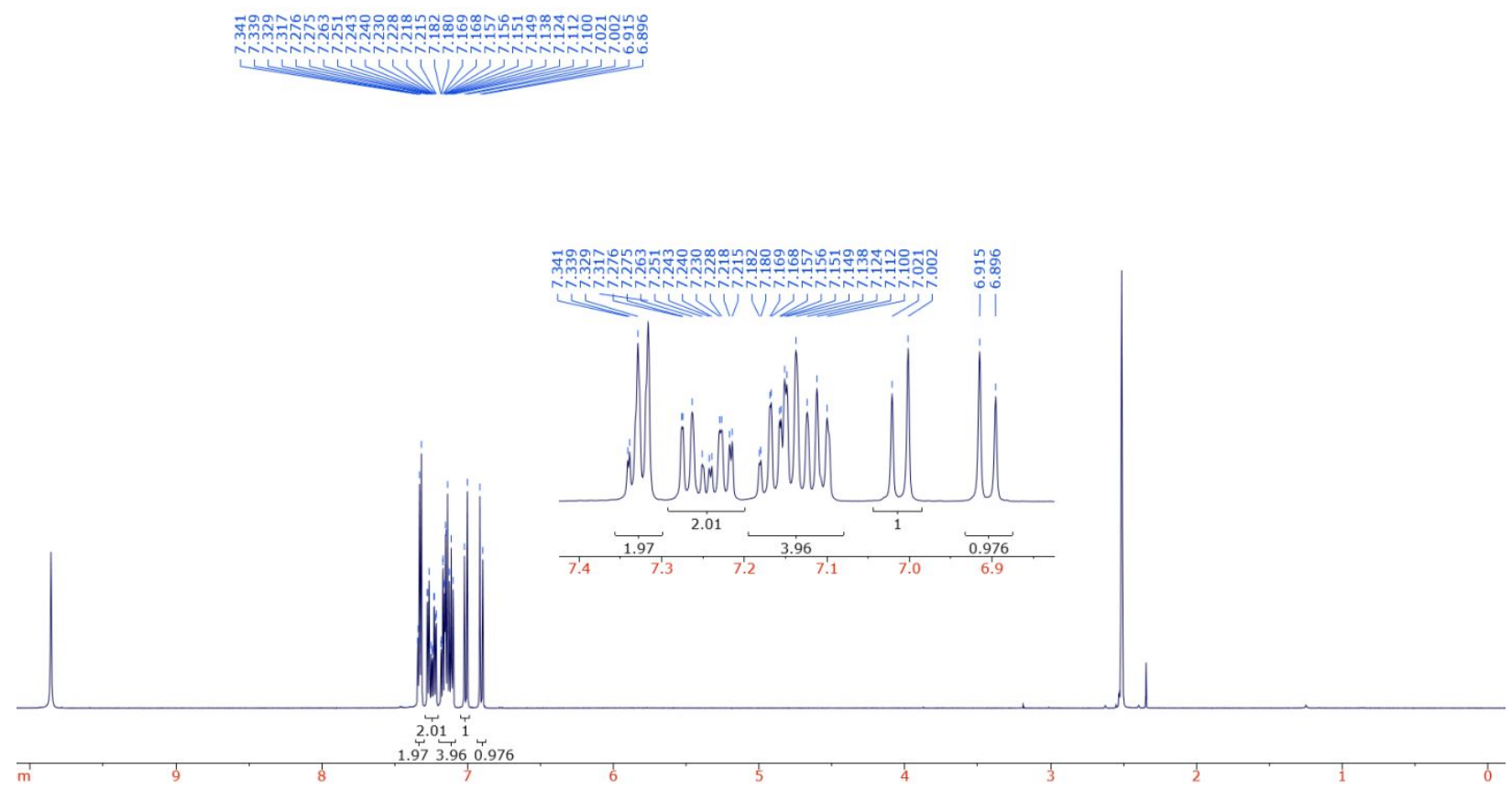

Figure S2. ${ }^{1} \mathrm{H}$ NMR spectrum of Compound 3 in $\mathrm{CDCl}_{3}$

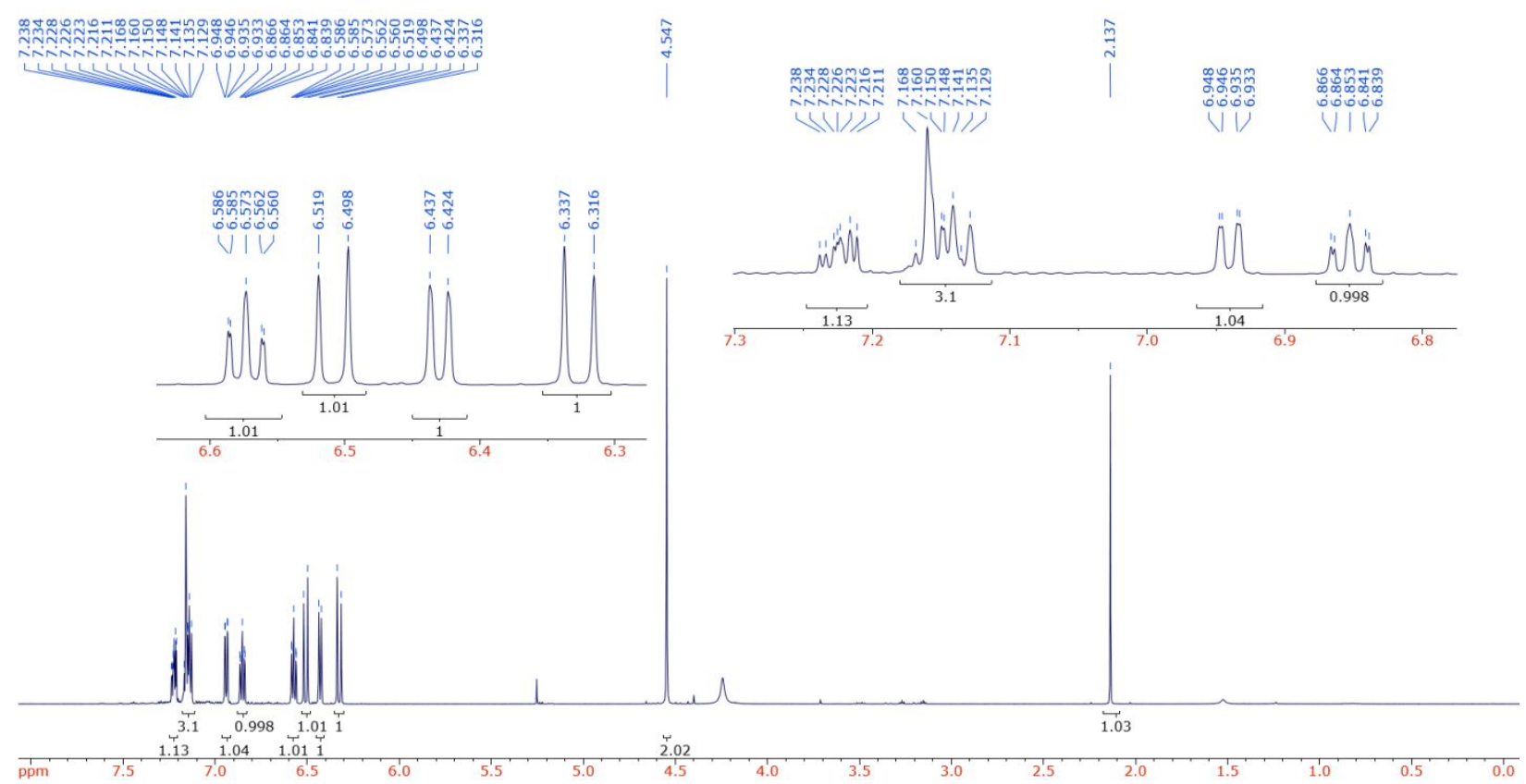

Figure S3. ${ }^{1} \mathrm{H}$ NMR spectrum of Compound 4 in $\mathrm{CDCl}_{3}$ 


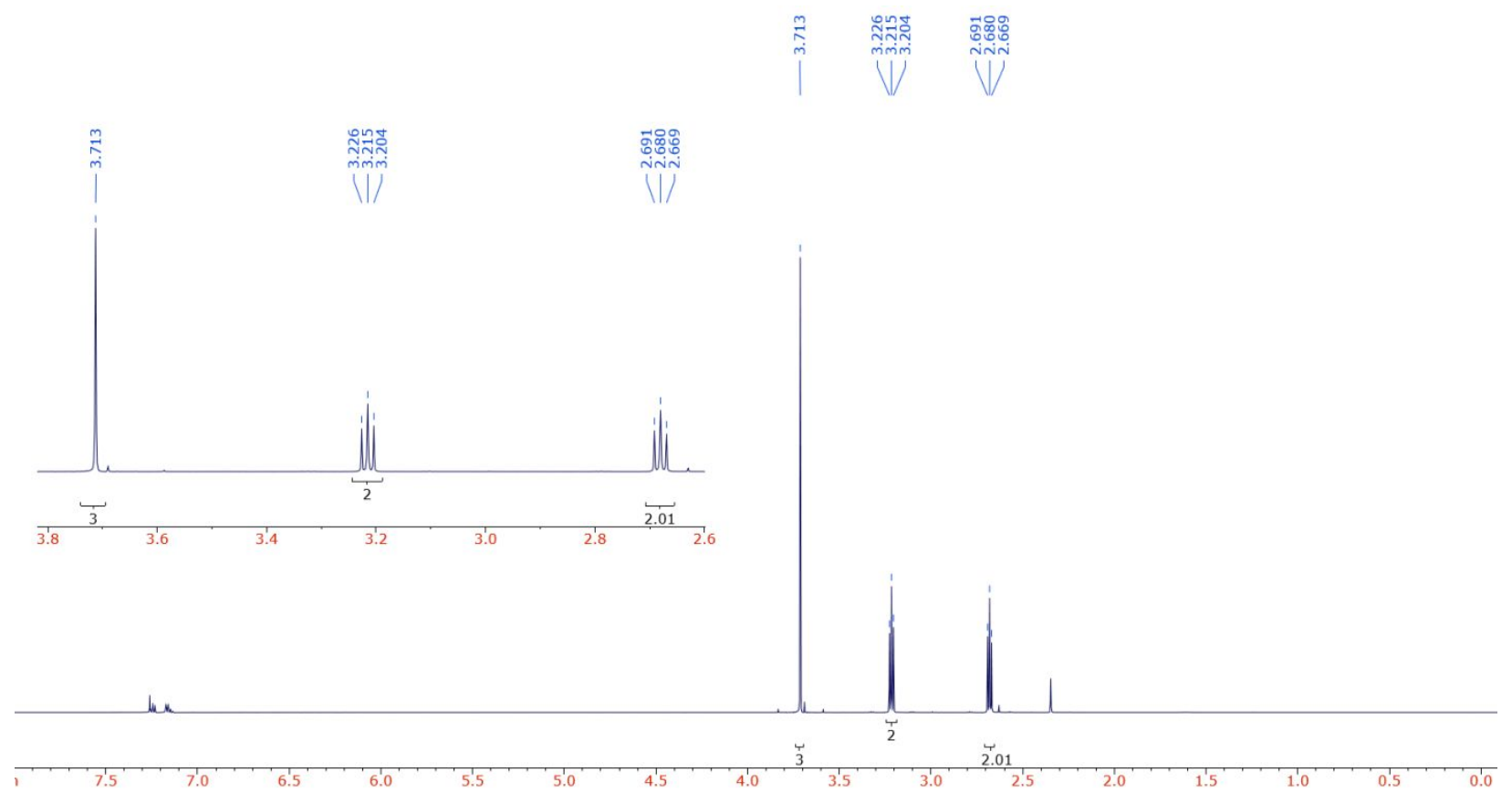

Figure S4. ${ }^{1} \mathrm{H}$ NMR spectrum of Compound 5 in $\mathrm{CDCl}_{3}$

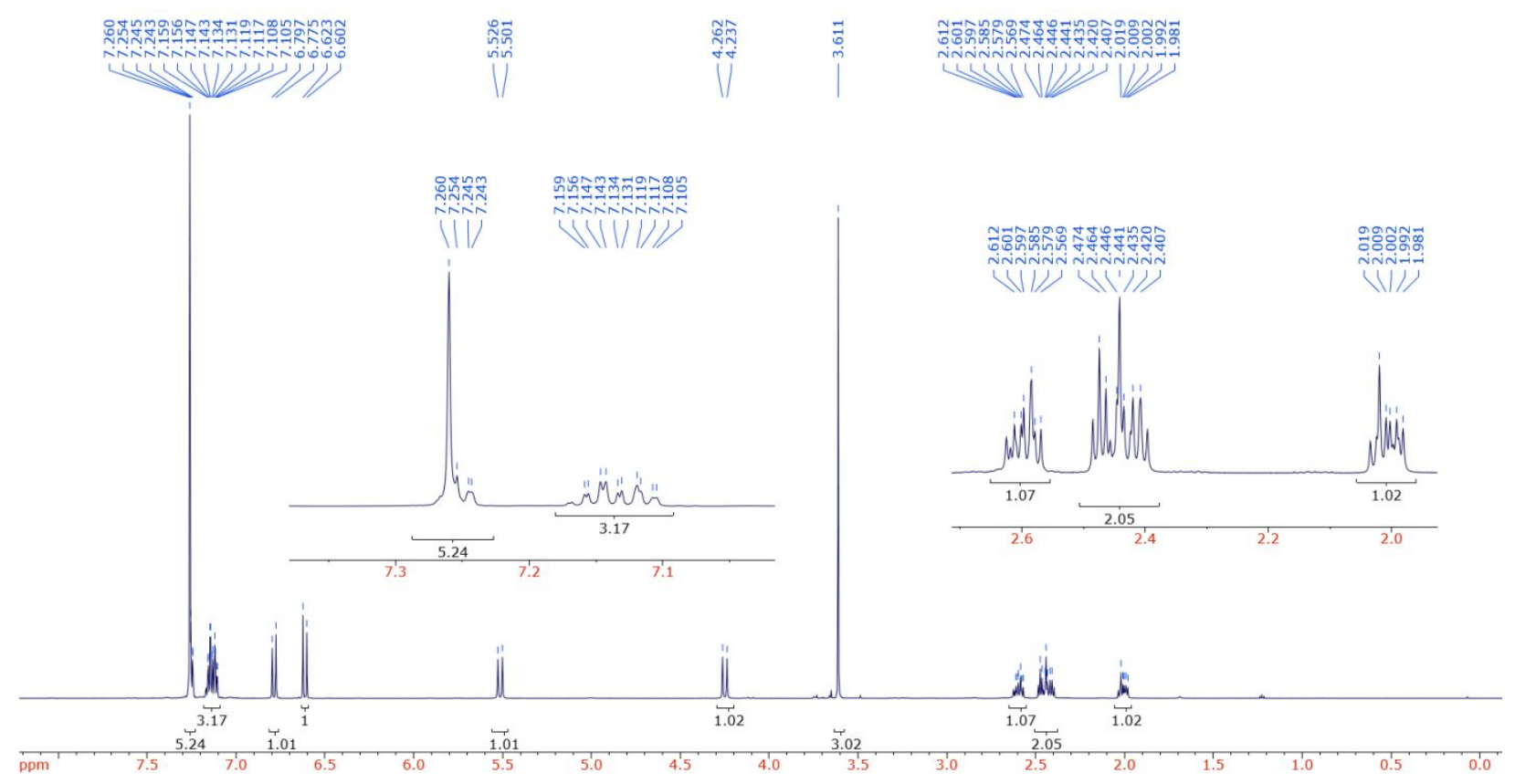

Figure S5. ${ }^{1} \mathrm{H}$ NMR spectrum of Compound 6 in $\mathrm{CDCl}_{3}$ 


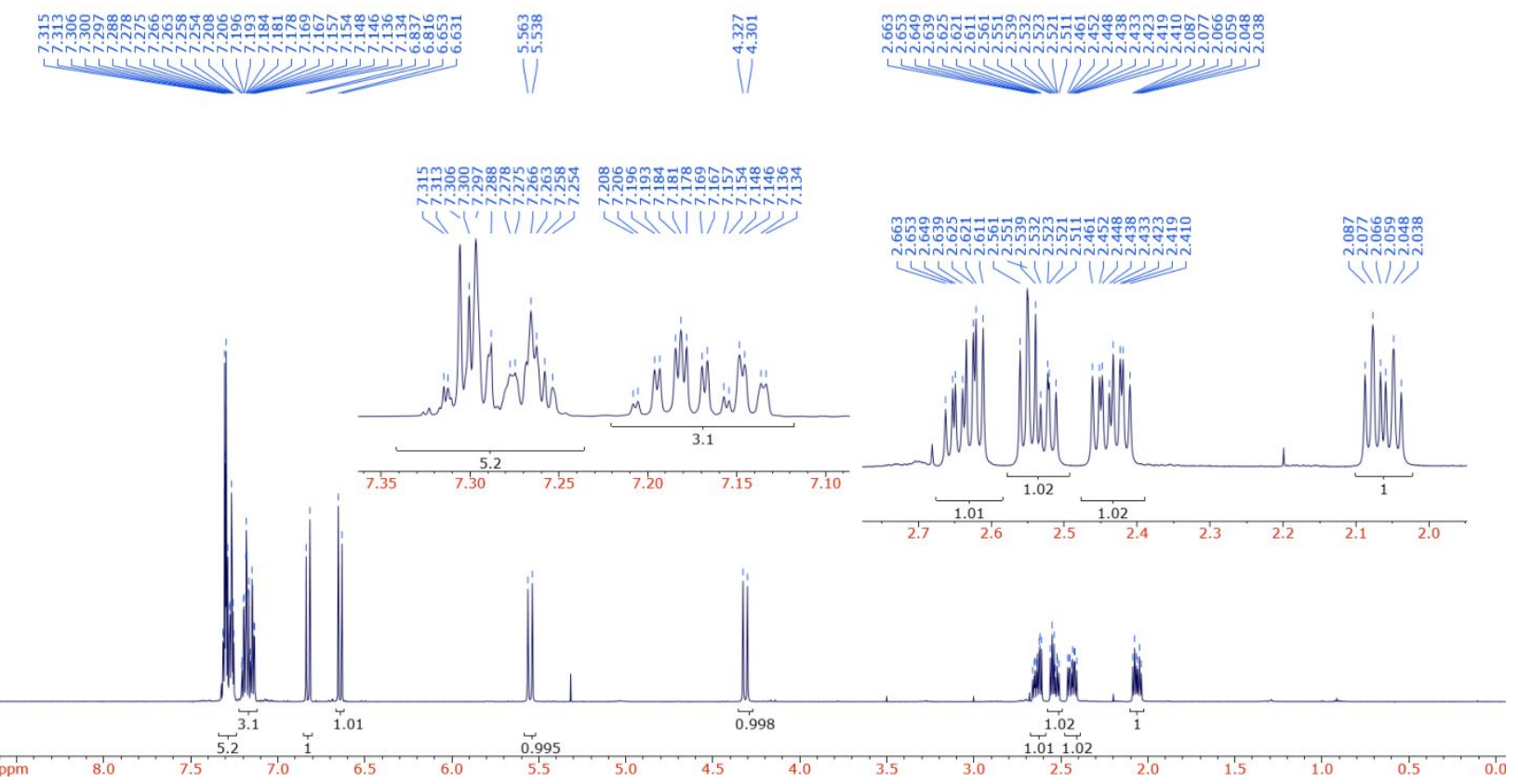

Figure S6. ${ }^{1} \mathrm{H}$ NMR spectrum of Compound 7 in $\mathrm{CDCl}_{3}$

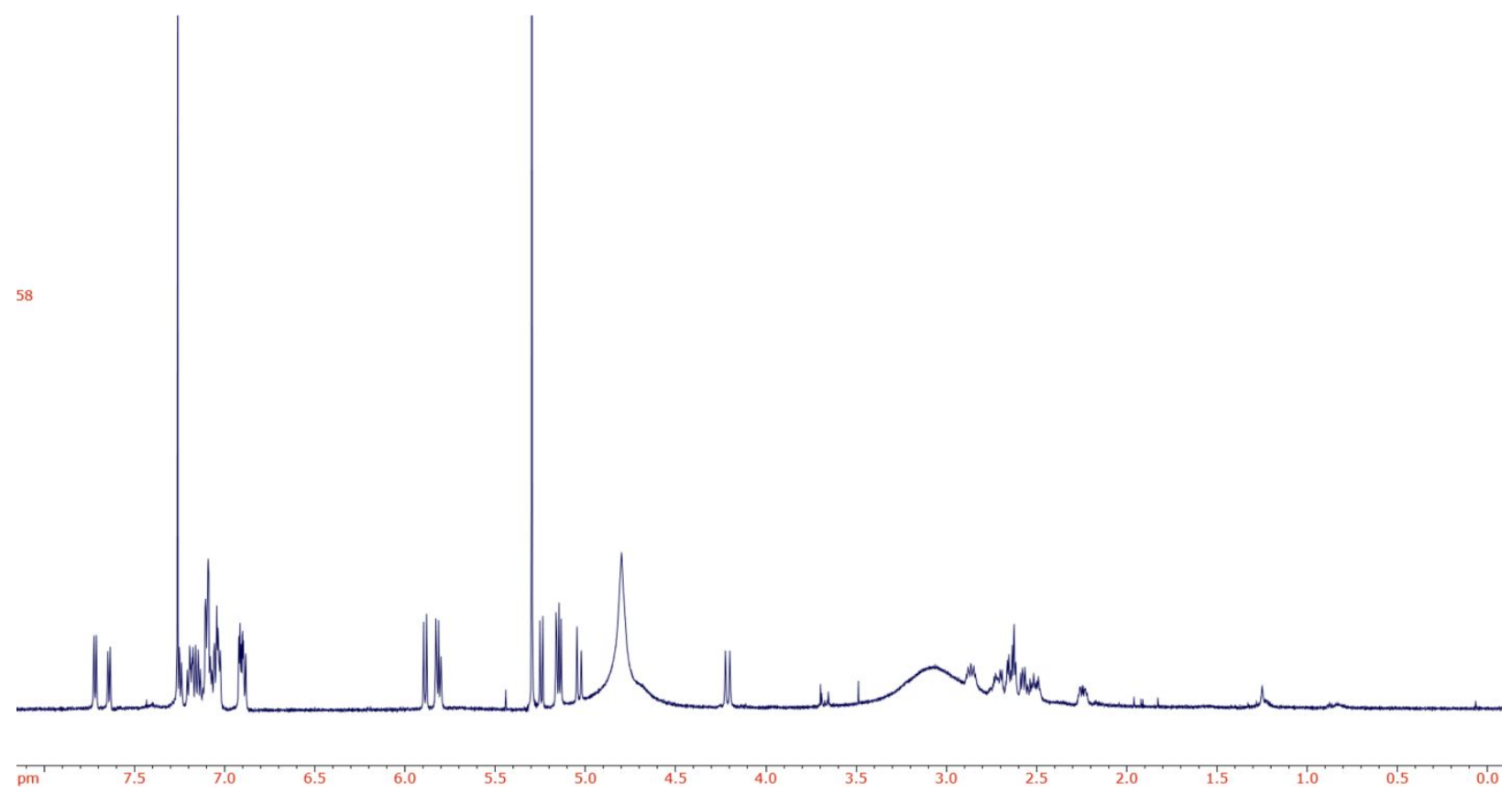

Figure S7. ${ }^{1} \mathrm{H}$ NMR spectrum of Compound 8 in $\mathrm{CDCl}_{3}$ (crude) 

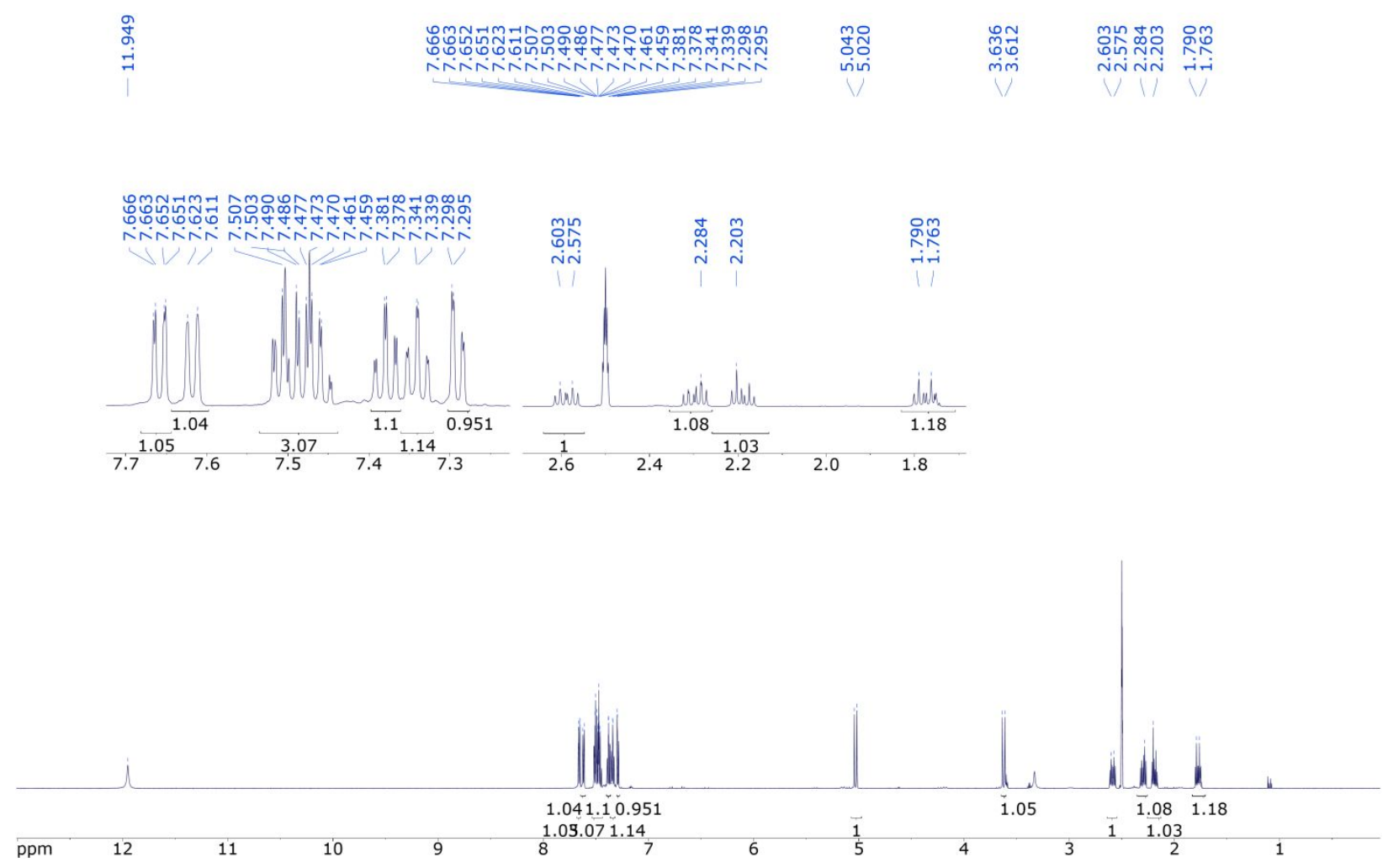

Figure S8. ${ }^{1} \mathrm{H}$ NMR spectrum of Compound 9 in DMSO- $d_{6}$

\section{HPLC Traces}

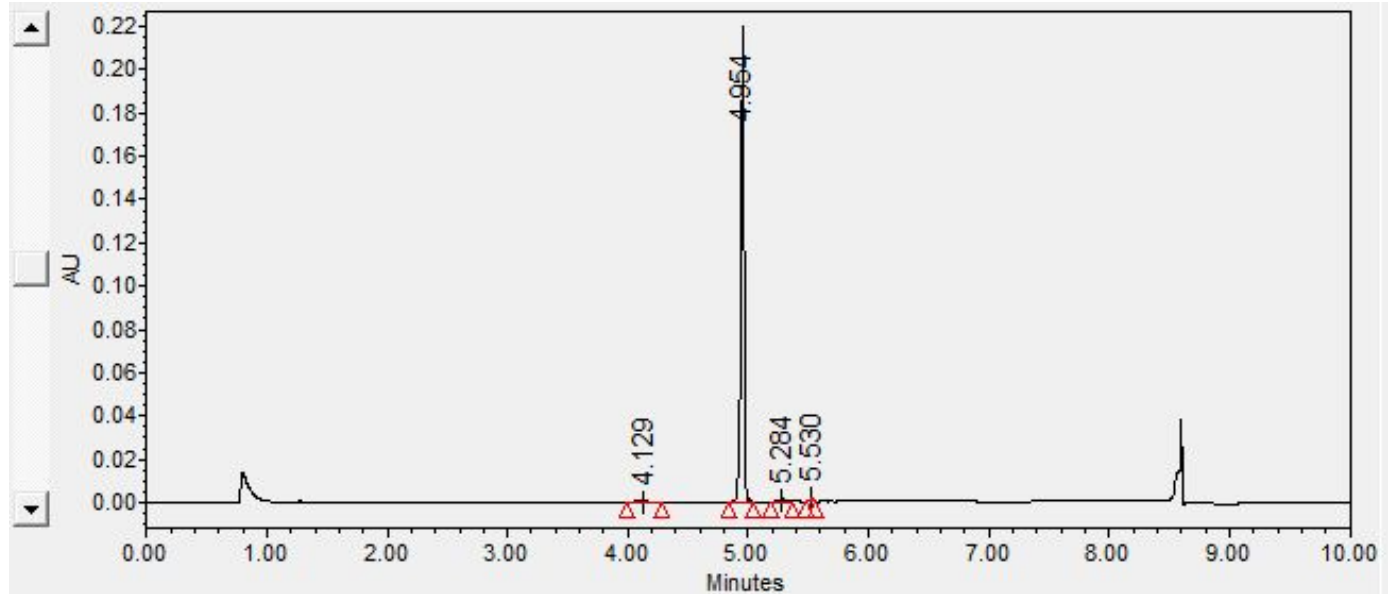

\begin{tabular}{|c|c|c|c|c|c|c|c|c|c|c|c|c|}
\hline E & Name & \begin{tabular}{|c|}
$\begin{array}{c}\text { Retention Time } \\
\text { (min) }\end{array}$ \\
\end{tabular} & $\%$ Area & Peak Codes & Dist Name & \begin{tabular}{|c|}
$\mathrm{Mn}$ \\
(Daltons)
\end{tabular} & \begin{tabular}{|c|} 
Mw \\
(Daltons)
\end{tabular} & \begin{tabular}{|c|} 
MP \\
(Daltons)
\end{tabular} & \begin{tabular}{|c|}
$\mathrm{Mv}$ \\
(Daltons)
\end{tabular} & \begin{tabular}{|c|}
$\mathrm{Mz}$ \\
(Daltons)
\end{tabular} & \begin{tabular}{|c|}
$\begin{array}{c}\mathrm{Mz}+1 \\
\text { (Daltons) }\end{array}$ \\
\end{tabular} & Polydis: \\
\hline 1 & & 4.129 & 1.79 & & & & & & & & & \\
\hline 2 & & 4.954 & 95.99 & & & & & & & & & \\
\hline 3 & & 5.284 & 1.36 & & & & & & & & & \\
\hline 4 & & 5.530 & 0.86 & & & & & & & & & \\
\hline
\end{tabular}

Figure S9. HPLC Trace of Compound 2 collected using HPLC method 1 


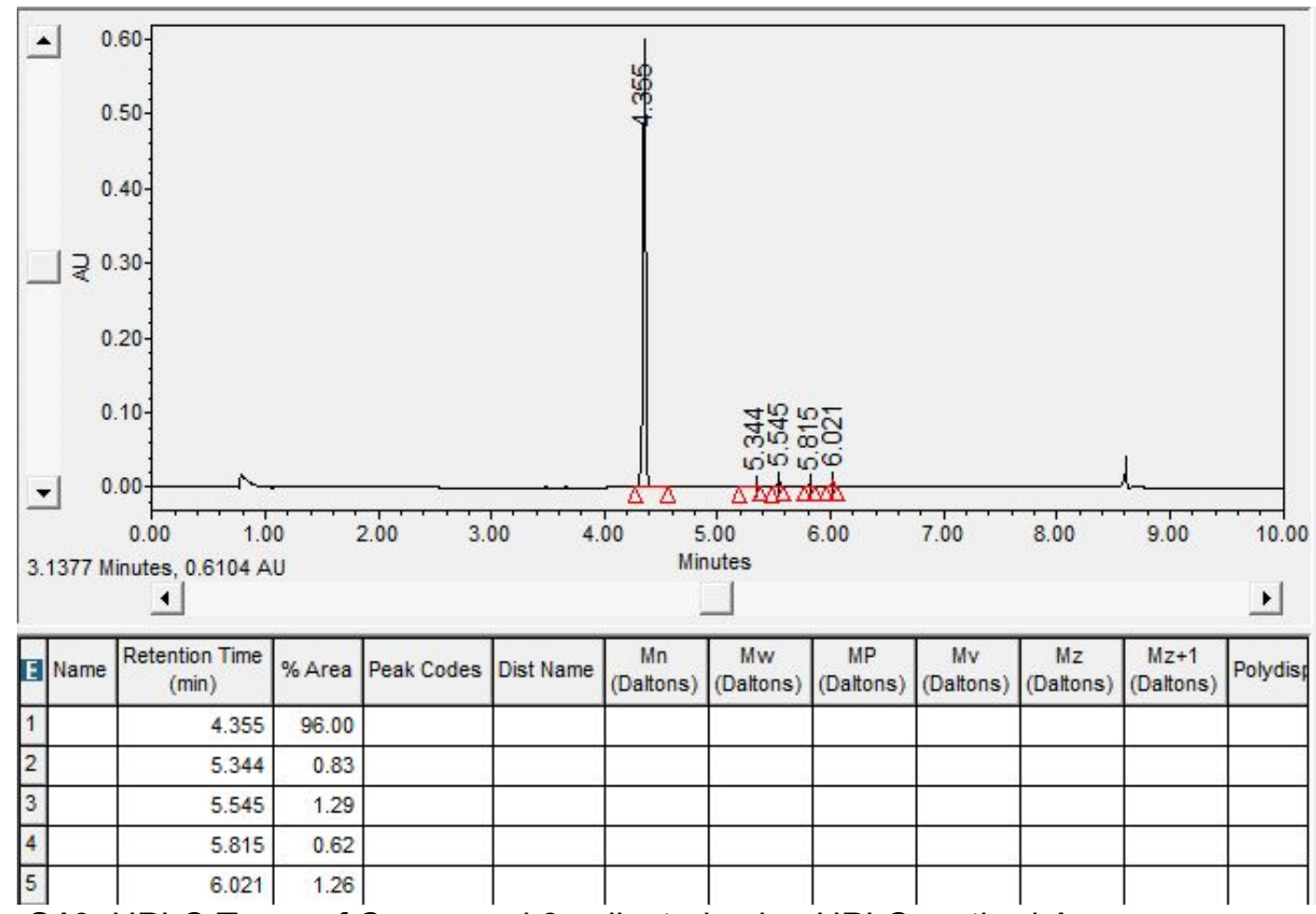

Figure S10. HPLC Trace of Compound 3 collected using HPLC method 1

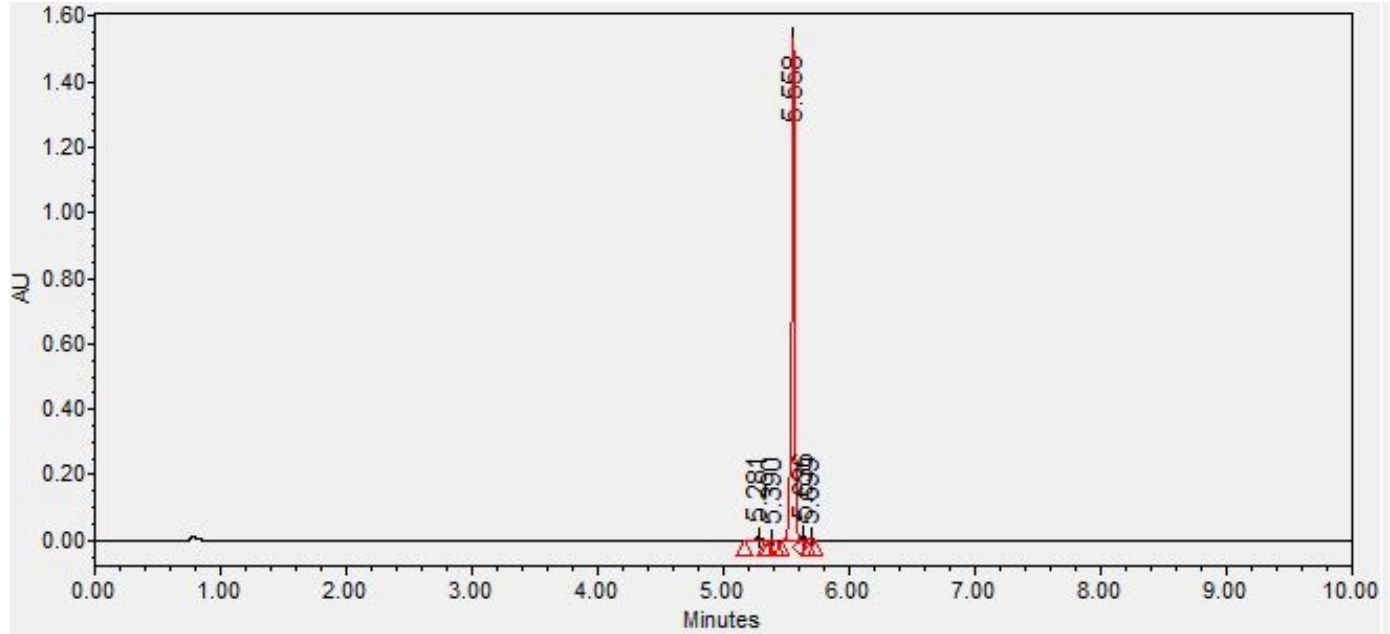

1

\begin{tabular}{|c|c|c|c|c|c|c|c|c|c|c|c|}
\hline ame & $\begin{array}{l}\text { Retention Time } \\
\text { (min) }\end{array}$ & $\%$ Area & Peak Codes & Dist Name & $\begin{array}{c}\text { Mn } \\
\text { (Daltons) }\end{array}$ & $\begin{array}{c}\text { Mw } \\
\text { (Daltons) }\end{array}$ & $\begin{array}{c}\text { MP } \\
\text { (Daltons) }\end{array}$ & $\begin{array}{c}\text { Mv } \\
\text { (Daltons) }\end{array}$ & $\begin{array}{c}\mathrm{Mz} \\
\text { (Daltons) }\end{array}$ & $\begin{array}{c}\text { Mz+1 } \\
\text { (Daltons) }\end{array}$ & Polydispersity \\
\hline & 5.281 & 0.94 & & & & & & & & & \\
\hline & 5.390 & 0.07 & & & & & & & & & \\
\hline & 5.558 & 97.76 & & & & & & & & & \\
\hline & 5.636 & 0.99 & & & & & & & & & \\
\hline & 5.699 & 0.24 & & & & & & & & & \\
\hline
\end{tabular}

Figure S11. HPLC Trace of Compound 4 collected using HPLC method 1 


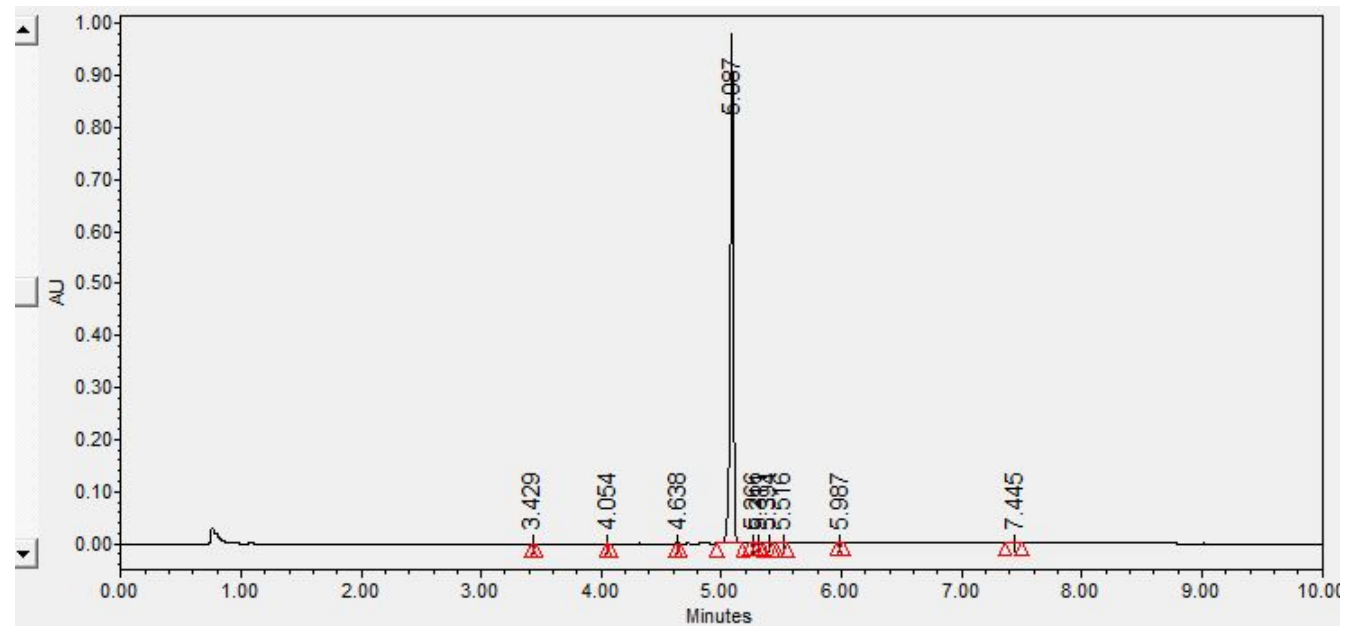

1

\begin{tabular}{|c|c|c|c|c|c|c|c|c|c|c|c|c|c|c|}
\hline & \begin{tabular}{|l|} 
Name \\
\end{tabular} & \begin{tabular}{|c|}
$\begin{array}{c}\text { Retention Time } \\
(\mathrm{min})\end{array}$ \\
\end{tabular} & $\%$ Area & Peak Codes & Dist Name & \begin{tabular}{|c|}
$\begin{array}{c}\text { Mn } \\
\text { (Daltons) }\end{array}$ \\
\end{tabular} & $\begin{array}{c}\mathrm{Mw} \\
\text { (Daltons) } \\
\end{array}$ & \begin{tabular}{|c|} 
MP \\
(Daltons)
\end{tabular} & \begin{tabular}{|c} 
Mv \\
(Daltons)
\end{tabular} & $\begin{array}{c}\mathrm{Mz} \\
\text { (Daltons) }\end{array}$ & $\begin{array}{c}\mathrm{Mz+1} \\
\text { (Daltons) }\end{array}$ & Polydispersity & \begin{tabular}{|l|}
{$[n] P$} \\
$(\mathrm{dVg})$ \\
\end{tabular} & $\begin{array}{c}\mathrm{K} \\
\mathrm{(dVg} \\
\end{array}$ \\
\hline$i$ & & 3.429 & 0.02 & & & & & & & & & & & \\
\hline 2 & & 4.054 & 0.02 & & & & & & & & & & & \\
\hline 3 & & 4.638 & 0.03 & & & & & & & & & & & \\
\hline 4 & & 5.087 & 99.34 & & & & & & & & & & & \\
\hline 5 & & 5.266 & 0.05 & & & & & & & & & & & \\
\hline 3 & & 5.311 & 0.03 & & & & & & & & & & & \\
\hline 7 & & 5.394 & 0.13 & & & & & & & & & & & \\
\hline 3 & & 5.516 & 0.18 & & & & & & & & & & & \\
\hline 3 & & 5.987 & 0.07 & & & & & & & & & & & \\
\hline$\overline{0}$ & & 7.445 & 0.13 & & & & & & & & & & & \\
\hline
\end{tabular}

Figure S12. HPLC Trace of Compound 6 collected using HPLC method 1

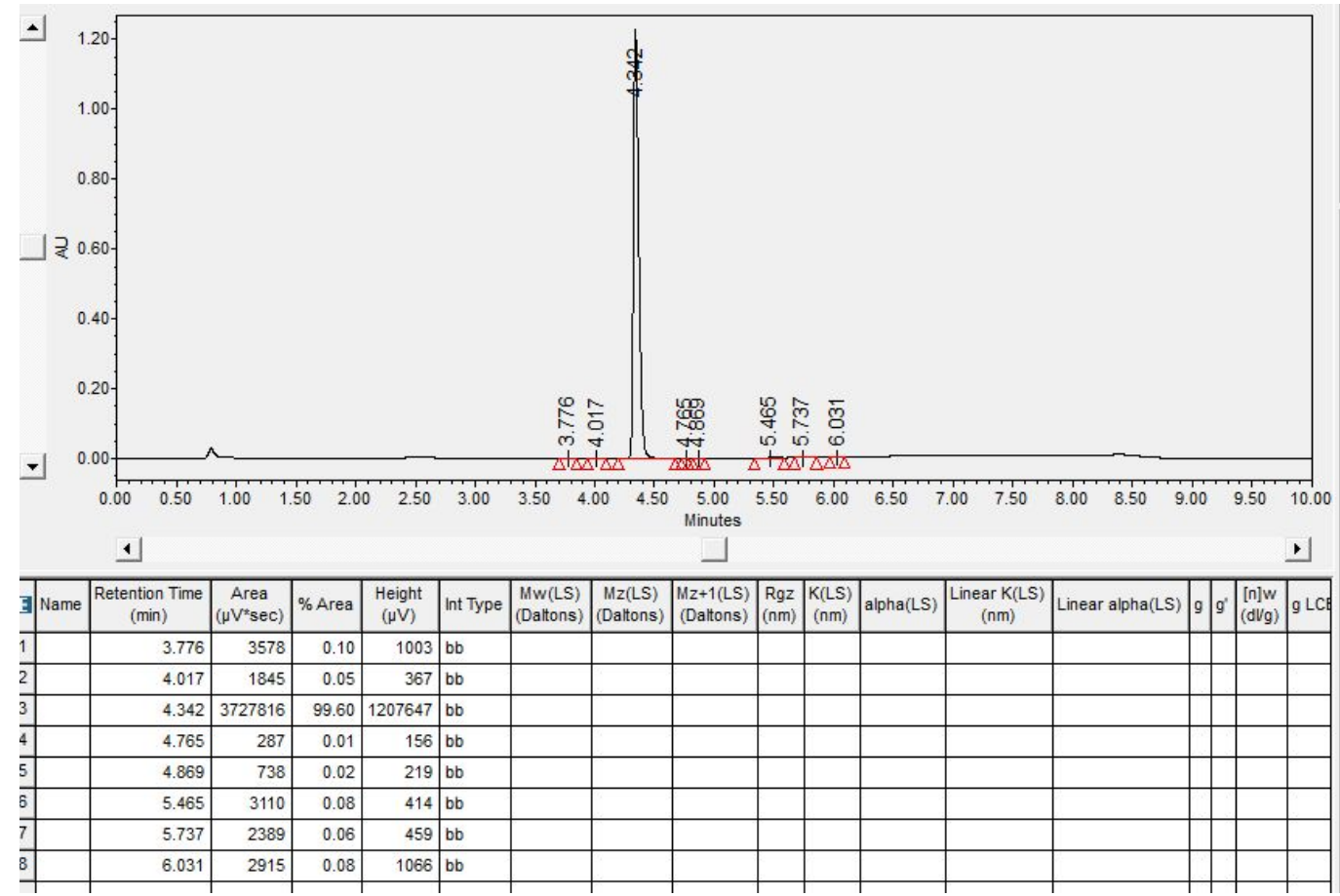

Figure S13. HPLC Trace of Compound 7 collected using HPLC method 1 


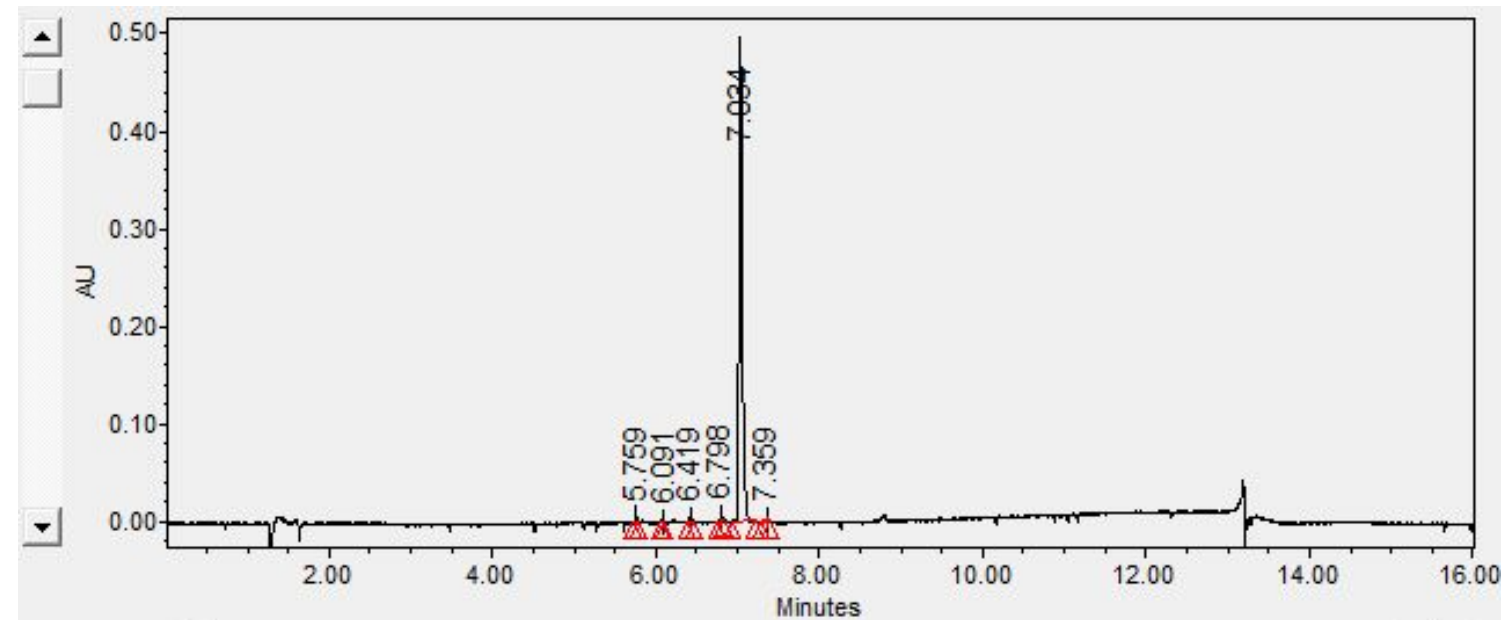

1

\begin{tabular}{l|r|r|r|r|r|l|l|l|l|l|l|l|l}
\hline E & Name & $\begin{array}{r}\text { Retention Time } \\
(\mathrm{min})\end{array}$ & $\begin{array}{c}\text { Area } \\
\left(\mu \mathrm{V}^{*} \mathrm{sec}\right)\end{array}$ & $\%$ Area & $\begin{array}{c}\text { Height } \\
(\mu \mathrm{V})\end{array}$ & Int Type & Amount & Units & Peak Type & Peak Codes & $\begin{array}{c}\text { MP } \\
(\text { Daltons })\end{array}$ & $\begin{array}{c}\text { Rg } \\
(\mathrm{nm})\end{array}$ & $\begin{array}{c}\mathrm{dn} / \mathrm{d} \\
(\mathrm{m} / \mathrm{g}\end{array}$ \\
\hline 1 & & 5.759 & 9009 & 0.65 & 4919 & $\mathrm{bb}$ & & & Unknown & & & & \\
\hline 2 & & 6.091 & 1461 & 0.10 & 950 & $\mathrm{bb}$ & & & Unknown & & & \\
\hline 3 & & 6.419 & 10362 & 0.74 & 4119 & $\mathrm{bb}$ & & & Unknown & & & & \\
\hline 4 & & 6.798 & 8140 & 0.58 & 4359 & $\mathrm{bb}$ & & & Unknown & & & & \\
\hline 5 & & 7.034 & 1354096 & 97.30 & 485354 & bb & & & Unknown & & & & \\
\hline 6 & & 7.359 & 8651 & 0.62 & 2967 & bb & & & Unknown & & & & \\
\hline
\end{tabular}

Figure S14. HPLC Trace of Compound 9 collected using HPLC method 2 